\title{
TWO REMARKS ON FIBER HOMOTOPY TYPE
}

\author{
John Milnor AND Edwin Spanier
}

Section 1 of this note considers the normal sphere bundle of a compact, connected, orientable manifold $M^{n}$ (without boundary) differentiably imbedded in euclidean space $R^{n+k}$. (These hypotheses on $M^{n}$ will be assumed throughout $\S 1$.) It is shown that if $k$ is sufficiently large then the normal sphere bundle has the fiber homotopy type of a product bundle if and only if there exists an $S$-map from $S^{n}$ to $M^{n}$ of degree one (i.e. for some $p$ there exists a continuous map of degree one from $S^{n+p}$ to the $p$-fold suspension of $M^{n}$ ). The proof is based on the fact that the Thom space of the normal bundle is dual in the sense of SpanierWhitehead [8] to the disjoint union of $M^{n}$ and a point.

Section 2 studies the tangent sphere bundle of a homotopy $n$-sphere. This has the fiber homotopy type of a product bundle if and only if $n$ equals 1,3 or 7 . The proof is based on Adams' work [1].

If $X$ is a space, $S^{k} X$ will denote the $k$-fold suspension of $X$ as in $[8,9]$. If $X$ has a base point $x_{0}$, then $S_{0}^{k} X$ will denote the $k$-fold reduced suspension and is the identification space $S^{k} X / S^{k} x_{0}$ obtained from $S^{k} X$ by collapsing $S^{k} x_{0}$ to a point (to be used as base point for $S_{0}^{k} X$ ). There is a canonical homeomorphism $S_{0}^{k} X \approx S^{k} \times X / S^{k} \vee X$.

Two fiber bundles with the same fiber and with projections $p_{1}: E_{1} \rightarrow$ $B, p_{2}: E_{2} \rightarrow B$ have the same fiber homotopy type $[3,4,10]$ if there exist fiber preserving maps $f_{i}: E_{i} \rightarrow E_{3-i}$ and fiber preserving ${ }^{1}$ homotopies $h_{i}: E_{i} \times I \rightarrow E_{i}$ such that $h_{i}(x, 0)=f_{3-i} f_{i}(x), h_{i}(x, 1)=x$.

Let $\xi$ denote an oriented $(k-1)$-sphere bundle. The total space of $\xi$ will be denoted by $\dot{E}$ and the total space of the associated $k$-disk bundle will be denoted by $E$. The Thom space $T(\xi)$ is the identification space $E / \dot{E}$ obtained from $\dot{E}$ by collapsing $\dot{E}$ to a single point (to be used as base point for $T(\xi)$ ). The following are easily verified:

(A) If $\xi_{1}, \xi_{2}$ are $(k-1)$-sphere bundles of the same fiber homotopy type, then $T\left(\xi_{1}\right), T\left(\xi_{2}\right)$ have the same homotopy type.

(B) If $\xi$ is a product bundle, then $T(\xi)$ is homeomorphic to $S_{0}^{k}\left(B \cup p_{0}\right)$ (where $B \cup p_{0}$ is the disjoint union of $B$ and a point, $p_{0}$, which is taken as the base point of $B \cup p_{0}$ ).

1. The normal bundle. If $X$ and $Y$ are spaces we let $[X, Y]$ denote the s set of homotopy classes of maps of $X$ into $Y$ and we let

Received May 19, 1959. The authors were supported by the Sloan Foundation and by the United States Air Force (Contract No. AF 49(638)-393 monitored by the Air Force Office of Scientific Research), respectively, during the period when this paper was in preparation.

1 The phrase "fiber-preserving" means that $p_{3-i} f_{i}(x)=p_{i}(x)$ and $p_{i} h_{i}(x, t)=p_{i}(x)$. 
$\{X, Y\}$ denote the set of $S$-maps of $X$ into $Y$ as in [8]. Thus, $\{X, Y\}$ is defined to be the direct limit of the sequence

$$
[X, Y] \stackrel{S}{\longrightarrow}[S X, S Y] \stackrel{S}{\longrightarrow} \cdots \stackrel{S}{\longrightarrow}\left[S^{p} X, S^{p} Y\right] \stackrel{S}{\longrightarrow} \cdots
$$

There is a natural map

$$
\phi:[X, Y] \longrightarrow\{X, Y\}
$$

which assigns to every homotopy class $[f] \varepsilon[X, Y]$ the $S$-map $\{f\}$ represented by any map of $[f]$. The following gives a sufficient condition for $\phi$ to be onto $\{X, Y\}$.

Lemma 1. Let $Y$ be a k-connected $C W$-complex $(k \geq 1)$ and let $X$ be a finite $C W$-complex with ${ }^{2} H^{q}(X)=0$ for $q>2 k+1$. Then $\phi([X, Y])=$ $\{X, Y\}$.

Proof. It suffices to prove that under the hypotheses of the lemma the map $S:[X, Y] \rightarrow[S X, S Y]$ is onto $[S X, S Y]$ because then, for each $p \geq 0$, the map $S:\left[S^{n} X, S^{p} Y\right] \rightarrow\left[S^{p+1} X, S^{p+1} Y\right]$ is onto $\left[S^{p+1} X, S^{p+1} Y\right]$ (because $S^{p} Y$ is $(p+k)$-connected and $H^{q}\left(S^{p} X\right)=0$ for $q>2 k+p+1$ and $2(k+p)+1 \geq 2 k+p+1)$.

Choose base points $x_{0} \varepsilon X, y_{0} \varepsilon Y$ and let $[X, Y]^{\prime}$ denote the set of homotopy classes of maps $\left(X, x_{0}\right) \rightarrow\left(Y, y_{0}\right)$. Since $Y$ is simply-connected the natural map $[X, Y]^{\prime} \rightarrow[X, Y]$ is a $1-1$ correspondence. Since $X, Y$ are $C W$-complexes the collapsing maps $S X \rightarrow S_{0} X$ and $S Y \rightarrow S_{0} Y$ are homotopy equivalences (Theorem 12 of [11]) so there are 1-1 correspondences

$$
\left[S_{0} X, S_{0} Y\right] \approx\left[S_{0} X, S Y\right] \approx[S X, S Y] .
$$

Since $S_{0} Y$ is simply connected, we also have a 1-1 correspondence $\left[S_{0} X, S_{0} Y\right]^{\prime} \approx\left[S_{0} X, S_{0} Y\right]$. Hence, it suffices to show that $S_{0}\left([X, Y]^{\prime}\right)=$ $\left[S_{0} X, S_{0} Y\right]^{\prime}$.

Let $\Omega S_{0} Y$ denote the space of closed paths in $S_{0} Y$ based at $y_{0}$. There is a canonical 1-1 correspondence $\left[S_{0} X, S_{0} Y\right]^{\prime} \approx\left[X, \Omega S_{0} Y\right]^{\prime}$ and a natural imbedding $Y \subset \Omega S_{0} Y$ such that the map $S_{0}:[X, Y]^{\prime} \rightarrow\left[S_{0} X, S_{0} Y\right]^{\prime}$ corresponds to the injection (see $\S 9$ of [7])

$$
[X, Y]^{\prime} \longrightarrow\left[X, \Omega S_{0} Y\right]^{\prime} .
$$

Hence, it suffices to show this injection is onto or, equivalently, that the natural injection (without base point condition) $[X, Y] \rightarrow\left[X, \Omega S_{0} Y\right]$ is onto.

2 When no coefficient group appears explicitly in the notation for a homology or cohomology group it is to be understood that the coefficient group is the group of integers. In dimension 0 the groups will be taken reduced. 
Since $Y$ is $k$-connected it follows from the suspension theorem (see $\S 7$ of [9]) that

$$
S_{0}: \pi_{i}(Y) \longrightarrow \pi_{i+1}\left(S_{0} Y\right)
$$

is $1-1$ if $i \leq 2 k$ and is onto if $i \leq 2 k+1$. Since $S_{0}$ corresponds to the injection map $\pi_{i}(Y) \rightarrow \pi_{i}\left(\Omega S_{0} Y\right)$, this is equivalent to the statement that

$$
\pi_{i}\left(\Omega S_{0} Y, Y\right)=0 \text { for } i \leq 2 k+1 \text {. }
$$

Since $Y$ is simply-connected the groups $\pi_{i}\left(\Omega S_{0} Y, Y\right)$ form a simple system for every $i$. Now the groups $H^{i}\left(X ; \pi_{i}\left(\Omega S_{0} Y, Y\right)\right.$ ) vanish for every $i$ because for $i \leq 2 k+1$ the coefficient group vanishes while for $i>2 k+1$ the groups vanish because of the assumption on the cohomology of $X$. By Theorem 4.4.2 of [2] it follows that any map $X \rightarrow \Omega S_{0} Y$ is homotopic to a map $X \rightarrow Y$, completing the proof.

REMARK. If in Lemma 1 we assume that $H^{q}(X)=0$ for $q>2 k$, then a similar argument shows that $\phi$ is $1-1$, however we shall not need this result.

Let $M^{n} \subset R^{n+k}$ be as in the introduction (i.e. $M^{n}$ is a differentiably imbedded manifold which is compact, connected, orientable, and without boundary). The following result relates the normal bundle of $M^{n}$ to $M^{n}$ itself by means of duality.

LEMMA 2. Let $\xi$ be the normal $(k-1)$-sphere bundle of $M^{n}$ in $R^{n+k}$. Then the Thom space $T(\xi)$ is weakly $(n+k+1)$-dual to the disjoint union $M^{n} \cup p_{0}$.

Proof. Regard $S^{n+k}$ as the one point compactification of $R^{n+k}$. Let $E$ be a closed tubular neighborhood of $M^{n}$ and assume $E$ is contained in a large disk $D^{n+k}$. Then $\left(D^{n+k}\right.$-interior $E$ ) is a deformation retract of $R^{n+k}-M^{n}=S^{n+k}-\left(M^{n} \cup\right.$ (point at infinity)). Using standard homotopy extension properties and the contractibility of $D^{n+k}$ it follows that if $\dot{E}$ denotes the boundary of $E$ then

$$
T(\xi)=E / \dot{E}=D^{n+k} /\left(D^{n+k}-\text { interior } E\right)
$$

has the homotopy type of the suspension $S\left(D^{n+k}-\right.$ interior $\left.E\right)$. Since $\left(D^{n+k}\right.$ - interior $\left.E\right)$ is an $(n+k)$-dual of $M^{n} \cup$ (point at infinity), and the suspension of an $(n+k)$-dual is an $(n+k+1)$-dual, this completes the proof.

REMARK. Lemma 2 shows that the $S$-type of $T(\xi)$ depends only on that of $M^{n}$. If $k$ is sufficiently large this implies that the homotopy type of $T(\xi)$ depends only on that of $M^{n}$. This suggests the conjecture 
that the fiber homotopy type of the normal bundle of any manifold $M^{n} \subset R^{n+k}, k$ large, is completely determined by the homotopy type of $M^{n}$. A similar conjecture can be made for the tangent bundle.

THeOREM 1. Let $M^{n} \subset R^{n+k}$ be as before and assume that $H_{q}\left(M^{n}\right)=0$ for $q<r$ and that $k \geq \min (n-r+2$, 3). The following statements are equivalent:

(1) There is an S-map $\alpha \varepsilon\left\{S^{n}, M^{n}\right\}$ such that

$$
\alpha_{*}: H_{n}\left(S^{n}\right) \approx H_{n}\left(M_{n}\right) .
$$

(2) The normal sphere bundle of $M^{n} \subset R^{n+k}$ has the fiber homotopy type of a product bundle.

(3) The disjoint union $M^{n} \cup p_{0}$ is weakly $(n+k+1)$-dual to $S_{0}^{k}\left(M^{n} \cup p_{0}\right)$.

Proof. (1) $\Rightarrow(2)$. Let $N$ denote the complement in $S^{n+k}$ of an open tubular neighborhood of $M^{n}$. Then $N$ is $(n+k)$-dual to $M^{n}$. The $S$-map $\alpha$ is $(n+k)$-dual to an $S$-map $\beta \varepsilon\left\{N, S^{k-1}\right\}$ such that $\beta^{*}: H^{k-1}\left(S^{k-1}\right) \approx H^{k-1}(N)$. Since $H^{p}(N) \approx H_{n+k-p-1}\left(M^{n}\right)$, we see that $H^{p}(N)=0$ if $p>n+k-r-1$. Since $S^{k-1}$ is $(k-2)$-connected, $k-2 \geq 1$, and $k \geq n-r+2$ (so $2(k-2)+$ $1 \geq n+k-r-1)$, it follows from Lemma 1 that there is a map $f: N \rightarrow S^{k-1}$ representing $\beta$. Then $f^{*}: H^{k-1}\left(S^{k-1}\right) \approx H^{k-1}(N)$. Let $\dot{E}$ be the boundary of $N$ (so $\dot{E}$ is the normal $(k-1)$-sphere bundle of $M^{n}$ ), and let $F$ be a fiber of $\dot{E}$. Then the inclusion map $F \subset N$ induces an isomorphism $H^{k-1}(N) \approx H^{k-1}(F)$ (because by Corollaries III. 15 and I.5 of [10] or by Theorems 14 and 21 of [5] we have $H^{k-1}(\dot{E}) \approx H^{k-1}\left(M^{n}\right)+Z$ and the injection $H^{k-1}(N) \rightarrow H^{k-1}(\dot{E})$ maps isomorphically onto the second summand while the injection $H^{k-1}(\dot{E}) \rightarrow H^{k-1}(F)$ maps the second summand isomorphically.) Therefore, the map $f \mid \dot{E}: \dot{E}_{-\rightarrow S^{k-1}}$ has the property that its restriction to a fiber $F$ induces an isomorphism of the cohomology of $S^{k-1}$ onto that of $F$ so is a homotopy equivalence of $F$ with $S^{k-1}$. This implies (by Corollary 2 on p. 121 of [3]) that $\dot{E}$ has the same fiber homotopy type as a product bundle.

$(2) \Rightarrow(3)$. By Lemma $2, T(\xi)$ is weakly $(n+k+1)$-dual to $M^{n} \cup p_{0}$. If $\xi$ is of the same fiber homotopy type as a product bundle, it follows from $(A),(B)$ that $T(\xi)$ is of the same homotopy type as $S_{0}^{k}\left(M^{n} \cup p_{0}\right)$. Combining these two statements gives the result.

$(3) \Rightarrow(1)$ assume $M^{n} \cup p_{0}$ is weakly $(n+k+1)$-dual to $S_{0}^{k}\left(M^{n} \cup p_{0}\right)$. The map $M^{n} \cup p_{0} \rightarrow S^{0}$ collapsing each component of $M^{n} \cup p_{0}$ to a single point represents an $S$-map $\beta: S_{0}^{k}\left(M^{n} \cup p_{0}\right) \rightarrow S_{0}^{k}\left(S^{0}\right)=S^{k}$ such that $\beta^{*}: H^{k}\left(S^{k}\right) \approx H^{k}\left(S_{0}^{k}\left(M^{n} \cup p_{0}\right)\right)$. By duality there is an $S$-map $\alpha \varepsilon\left\{S^{n}\right.$, $\left.M^{n} \cup p_{0}\right\}$ such that $\alpha_{*}: H_{n}\left(S^{n}\right) \approx H_{n}\left(M^{n} \cup p_{0}\right) \approx H_{n}\left(M^{n}\right)$. Since. 


$$
\left\{S^{n}, M^{n} \cup p_{0}\right\} \approx\left\{S^{n}, M^{n}\right\}+\left\{S^{n}, S^{0}\right\},
$$

the result is proved.

As a corollary we obtain the following result proved by Massey [4].

CoRollary. Let $M^{n}$ be a homology sphere. Then the normal bundle of $M^{n}$ in $R^{n+k}$ has the same fiber homotopy type as a product bundle.

Proof. Since $r=n$, the case $k \geq 3$ follows from the theorem. For the cases $k=1,2$ it is well known that the normal bundle is, in fact, trivial.

Remark. Puppe [6] calls a manifold "sphere-like" if the unstable group $\pi_{n+1}\left(S M^{n}\right)$ contains an element of degree one. (The group $\pi_{n}\left(M^{n}\right)$ can contain an element of degree one if and only if $M^{n}$ is a homotopy sphere.) Theorem 1 shows that the normal sphere bundle of a spherelike manifold $M^{n} \subset R^{n+k}$ has the fiber homotopy type of a product bundle provided $k$ is sufficiently large. An example of a manifold with trivial normal bundle which is not sphere-like is provided by the real projective 3-space.

2. The tangent bundle. Let $M^{n}$ be as above (i.e. compact, connected, orientable, and without boundary), but let $E$ denote a closed tubular neighborhood of the diagonal in $M^{n} \times M^{n}$. If the tangent bundle has the fiber homotopy type of a product bundle, then there exists a map $\dot{E} \rightarrow S^{n-1}$ (where $\dot{E}$ is the boundary of $E$ ) having degree one on each fiber. This gives rise to a map $(E, \dot{E}) \rightarrow\left(D^{n}, S^{n-1}\right) \rightarrow\left(S^{n}\right.$, point $)$ of degree one and, hence, to a map

$$
M^{n} \times M^{n} \longrightarrow M^{n} \times M^{n} /\left(M^{n} \times M^{n} \text {-interior } E\right)=E / \dot{E} \longrightarrow S^{n}
$$

which has degree $(1,1)$ (the degree is $(1,1)$ because a generator of $H^{n}\left(S^{n}\right)$ maps, under the homomorphism induced by the above composite, into a cohomology class of $M^{n} \times M^{n}$ dual under Poincaré duality to the diagonal class of $\left.H_{n}\left(M^{n} \times M^{n}\right)\right)$.

Theorem 2. Suppose that $M^{n}$ has the homotopy type of an $n$-sphere. Then the tangent bundle has the fiber homotopy type of a product bundle if and only if $n$ equals 1,3 or 7 (and in this case the tangent bundle is a product bundle).

Proof. If a map $S^{n} \times S^{n} \rightarrow S^{n}$ of degree $(1,1)$ exists, then according to Adams $n$ must be equal to 1,3 or 7 (see Theorem la of [1]).

Conversely, if $n$ equals 1,3 or 7 then $\pi_{n-1}(S O(n))=0$. Using 
obstruction theory it follows that any homotopy $n$-sphere is parallelizable. This completes the proof.

\section{REFERENCES}

1. F. Adams, On the nonexistence of elements of Hopf invariant one, Bull. Amer, Math. Soc., 64 (1958), 279-282.

2. A. L. Blakers and W. S. Massey, The homotopy groups of a triad I, Ann. of Math., 53 (1951), 161-205.

3. A. Dold, Über fasernweise Homotopieäquivalenz von Faserräumen, Math. Zeits., 62 (1955), 111-136.

4. W. S. Massey, On the normal bundle of a sphere imbedded in Euclidean space (to appear).

5. J. Milnor, Lectures on characteristic classes (mimeographed), Princeton University, 1958.

6. D. Puppe, Homotopiemengen und ihre induzierten Abbildungen II, Math. Zeits., 69 (1958), 395-417.

7. E. Spanier, Infinite symmetric products, function spaces, and duality, Ann. of Math., 69 (1959), 142-198.

8. E. Spanier and J. H. C. Whitehead, Duality in homotopy theory, Mathematika, 2 (1955), $56-80$.

9. E. Spanier and J. H. C. Whitehead, The theory of carriers and S-theory, in Algebraic Geometry and Topology (a symposium in honor of S. Lefschetz), Princeton University Press, 1957, pp. 330-360.

10. R. Thom, Espaces fibrés en sphères et carrés de Steenrod, Ann. Ecole Norm. Sup., 69 (1952), 109-182.

11. J. H. C. Whitehead, Combinatorial Homotopy I, Bull, Amer. Math. Soc., 55 (1949), $213-245$.

PRINCETON UNIVERSITY

UNIVERSITY OF CHICAGO AND

The INSTitute FOR AdVANCED StUdy 